\title{
Nutrição e saúde infantil em uma comunidade indígena Teréna, Mato Grosso do Sul, Brasil
}

\author{
Child health and nutrition in a Teréna indigenous \\ community, Mato Grosso do Sul, Brazil
}

Dulce Lopes Barboza Ribas 1

Alfredo Sganzerla 2

José Roberto Zorzatto 3

Sonia Tucunduva Philippi 4

1 Departamento de

Tecnologia de Alimentos e Saúde Pública, Centro de Ciências Biológicas e da Saúde, Universidade Federal de Mato Grosso do Sul.

Rua Itiquira 247, Campo Grande, $M S$ 79021-290, Brasil.

dbribas@zaz.com.br

2 Universidade Católica Dom Bosco. Av. Tamandaré 6000, Campo Grande, MS 79117-010 Brasil.

freialfredo@enersulnet.com.br

3 Departamento de

Computação e Estatística,

Centro de Ciências

Exatas e Tecnológicas,

Universidade Federal

de Mato Grosso do Sul.

Rua Gonçalves Dias 72 ,

Campo Grande, MS

79004-200, Brasil.

zorzatto@dct.ufms.br

4 Departamento de Nutrição,

Faculdade de Saúde

Pública, Universidade

de São Paulo.

Av. Dr. Arnaldo 715,

São Paulo, SP

01246-904, Brasil.

philippi@usp.br

\begin{abstract}
This paper describes the health and nutritional situation of South American Indian children from a Teréna community, characterizing their nutritional status, food consumption, and socioeconomic and environmental conditions. The sample included 100 children, ranging from 0 to 59 months of age and living in Aldeia Córrego do Meio, Mato Grosso do Sul. Prevailing nutritional deficits were: $8.0 \%$ for the weight-for-age index, $16.0 \%$ for height-for-age, and 5.0\% for weight-for-height. The growth deficit rate was higher than that of the Brazilian population as a whole, probably reflecting the precarious socioeconomic, environmental, and health conditions in this Teréna community. Analysis of the average nutrient sufficiency in the infant diet showed that nutritional recommendations for the different groups were not complied with. New studies, characterized as transdisciplinary and longitudinal, are necessary to better understand this process.
\end{abstract}

Key words Anthropometry; Child Health; Nutritional Status; Teréna; South American Indians

Resumo O trabalho descreve as condições de saúde e nutrição de crianças indígenas Teréna, caracterizando o estado nutricional infantil, o consumo de alimentos, as condições sócio-econômicas e ambientais. Foi estudada uma amostra de 100 crianças de 0 a 59 meses, residentes na Aldeia Córrego do Meio, Mato Grosso do Sul, Brasil. As prevalências de déficits nutricionais determinadas foram de 8,0\% para o índice peso-para-idade, de 16\% para o índice estatura-para-idade e, para obesidade, de 5\%. A proporção de retardo do crescimento observada é superior à encontrada na população brasileira como um todo, provavelmente por refletir as precárias condições sócio-econômicas, ambientais e de saúde em que vive esta comunidade Teréna. A análise da adequação percentual média dos nutrientes que compõem a dieta infantil indicou o não atendimento às recomendações nutricionais nas diferentes faixas etárias. Novos estudos de caráter multidisciplinar e longitudinal são necessários para melhor entendimento dos processos identificados.

Palavras-chave Antropometria; Saúde Infantil; Estado Nutricional; Teréna; Índios Sul-Americanos 


\section{Introdução}

Ampliar as possibilidades de observação e construção de indicadores sociais e biológicos de grupos indígenas é uma necessidade urgente, principalmente por instituições de ensino e pesquisa próximas geograficamente de áreas indígenas e com o compromisso de investigar e intervir no âmbito de realidades regionais.

A população deste estudo pertence ao subgrupo Guaná-Txané, originários do Chaco paraguaio, remanescentes da família Aruák. Dados sobre a migração inicial dos Teréna para o sul de Mato Grosso apontam o ano de 1649, quando os Mbayá-Guaykurú tornaram-se dominadores do território pantaneiro, submetendo outras nações indígenas, dentre elas a nação Guaná (Gadelha, 1982).

Seguindo a tradição de povo agricultor, as áreas cultivadas pelos Teréna circundavam a aldeia. A terra não gerava renda e servia basicamente para instalar moradias e produzir alimentos de subsistência. A unidade econômica era a família extensa, com atividades de plantio de roças, coleta de raízes e de frutos silvestres, combinadas com a caça e a pesca (Oberg, 1949; Silva, 1949).

A terra é um bem comum nas áreas Teréna, mas a organização da produção inclui divisões com posse diferencial da terra e dos meios de produção. As atividades de caça, pesca, coleta de raízes e frutos silvestres são realizadas esporadicamente, com dificuldades e limitações devido à redução da área na reserva, e os grupos domésticos são constituídos por famílias elementares com um casal e filhos, com minoria formada por dois a três casais e filhos.

Investigações desenvolvidas em diversas comunidades indígenas constituem importantes fontes de dados indispensáveis ao monitoramento de alterações, ficando cada vez mais evidente a necessidade de proceder-se a estudos de avaliação nutricional como forma de medir o papel exercido pelos fatores ambientais cristalizados nas condições de vida, saúde e nutrição de populações indígenas. Esta pesquisa teve por objetivo estimar as condições de saúde e nutrição de crianças indígenas Teréna, através da avaliação nutricional, análise da utilização de serviço de assistência materno-infantil, consumo alimentar e condições sócioeconômicas e ambientais.

\section{Métodos}

Este estudo epidemiológico - com base em observação transversal - foi realizado no período de julho a dezembro de 1999, em área indígena Terena denominada Aldeia Córrego do Meio, pertencente ao Município de Sidrolândia, Mato Grosso do Sul, Brasil.

Foram identificadas 66 famílias com crianças menores de cinco anos de idade; destas, 64 aceitaram participar do estudo. A população infantil nesta faixa etária totalizava 103 crianças, sendo estudadas $100(97,1 \%)$. Para a análise das variáveis referentes às condições sócioeconômicas e ambientais foram estudados 64 domicílios, com 59 pais e 64 mães.

As medidas antropométricas foram obtidas pelo coordenador da pesquisa, auxiliado por entrevistadores devidamente treinados. A técnica de antropometria adotada foi baseada em recomendações da Organização Mundial de Saúde e do documento Anthropometric Standardization Reference Manual (Lohman et al., 1988; WHO, 1995). Após a obtenção das medidas, os resultados eram fornecidos aos pais com a análise de adequação peso-para-idade, apresentada com preenchimento e interpretação do gráfico do cartão da criança, bem como com a valorização da participação ativa dos pais na vigilância alimentar e nutricional.

As medidas realizadas foram: massa corporal e comprimento ou estatura. Na medição do comprimento de crianças abaixo de 36 meses foi utilizado antropômetro de madeira, tipo horizontal, com régua graduada em centímetros, construído especialmente para a pesquisa. Para obtenção da estatura das crianças acima de 36 meses empregou-se antropômetro portátil eletrônico.

As crianças menores de 24 meses foram pesadas sem roupa, no colo dos pais. As crianças a partir de 24 meses foram avaliadas em balança digital eletrônica, estando descalças e usando roupas leves.

Considerou-se como atenção pré-natal todo o acompanhamento periódico e programado da gestante com o objetivo de prevenir possíveis complicações da gravidez, do parto e pós-parto, assim como de preparar a mãe para os cuidados com a criança. Foram determinados ainda o local de nascimento e o tipo de parto. $O$ peso ao nascer foi identificado pela informação da mãe, mediante comprovação no cartão da criança ou documento fornecido pela maternidade. Com relação à imunização foram considerados os dados confirmados na carteira de vacina ou senha de campanha e cicatriz vacinal. 
As análises do estado nutricional infantil foram realizadas a partir dos índices peso-paraidade (P/I), peso-para-estatura (P/E) e estatura-para-idade (E/I). Utilizou-se, como ponte de corte, dois escores $\mathrm{z}$ abaixo e acima da mediana da população de referência do National Center for Health Statistics (NCHS, 1978; WHO, 1995). O P/I analisa a relação entre o peso observado e o peso de referência para a idade e não discrimina o período em que ocorreu o déficit alimentar; mostra-se sensível para detectar alterações na massa corpórea, porém é pouco específico para a identificação de desnutrição a partir de observação única. $O$ índice $\mathrm{E} / \mathrm{I}$ avalia o crescimento linear, vulnerável às carências nutricionais crônicas e de longa duração - tais como alimentação continuamente deficiente e episódios repetidos de doenças infecciosas -, empregando-se o termo retardo de crescimento para descrever crianças com déficits de estatura-para-idade. A classificação de sobrepeso em crianças é baseada no índice $\mathrm{P} / \mathrm{E}$ com valores acima de +2 escores z. Estimou-se também o perímetro braquial - tendo, como ponto de corte, $-2 \mathrm{e}+2$ escores $\mathrm{z}-$, comparado com valores do I e II National Health and $\mathrm{Nu}$ trition Examination Survey (NHANES I e II) (WHO, 1995).

$\mathrm{Na}$ avaliação do consumo alimentar, a identificação da dieta da população infantil foi realizada por meio do método recordatório de 24 horas referente ao dia anterior à pesquisa, isto é, desde a primeira refeição da manhã até a última refeição da noite, calculando-se a ingestão calórica e a composição da dieta em proteínas, carboidratos, lipídios, cálcio, ferro, retinol e vitamina C. As informações foram obtidas em medidas caseiras e convertidas posteriormente em pesos e volumes. Como instrumento auxiliar no dimensionamento das porções consumidas foram utilizados utensílios usuais da área em estudo. Os instrumentos usados neste estudo passaram por pré-teste em uma comunidade indígena urbana, na qual 63 famílias Teréna foram entrevistadas.

A produção de alimentos na terra disponível, a distribuição interfamiliar dos alimentos, a prática da caça, pesca e a coleta de frutos silvestres foram investigados por intermédio de entrevista com os pais e lideranças e mediante observação no período de permanência na área, que cobriu parte da estação de seca - julho a outubro - e o início da estação das chuvas -novembro e dezembro. As dificuldades encontradas na obtenção dos dados de consumo alimentar infantil restringiram-se à variação ocorrida no consumo de alimentos nos dias de ida à cidade para aquisição dos gêneros ali- mentícios. Quanto à composição da dieta, os alimentos eram os mesmos utilizados pelos moradores de comunidades rurais da região, já do conhecimento dos entrevistadores, e as refeições seguiam horários relativamente regulares; a presença da guia indígena permitiu esclarecimentos sobre a forma de preparo, utensílios, e tamanho das porções. Outras informações coletadas - tais como: aleitamento materno, morbidade referida e consumo alimentar da família - necessitam ser estudadas em maior profundidade e serão descritas posteriormente em outro artigo.

Os dados referentes ao consumo de alimentos foram avaliados por meio do software Virtual Nutri, efetuando-se análises do valor quantitativo e qualitativo da dieta, além de sua adequação (Philippi et al., 1996). As médias do consumo de nutrientes foram comparadas às recomendações dietéticas do National Research Council (NRC, 1989).

Como a amostra estudada foi constituída por $97,1 \%$ das crianças, não foi utilizado procedimento de inferência estatística, uma vez que os parâmetros obtidos correspondem aos valores reais. Todas as informações foram conseguidas mediante entrevista, com registro em formulário próprio, sendo fornecidas pelos pais e muitas vezes complementadas pelos avós, que também participam ativamente do cuidado infantil.

Os trabalhos tiveram início após a obtenção de parecer favorável da Comissão Nacional de Ética em Pesquisa, autorização de ingresso em terra indígena e consentimento livre e esclarecido dos pais das crianças. Todos os domicílios foram visitados por dois membros da equipe e um guia indígena, indicado especialmente para o acompanhamento dos trabalhos. As visitas iniciais mostraram-se de fundamental importância para o conhecimento da dinâmica da comunidade e dos valores culturais, ademais de prestar esclarecimentos relativos à pesquisa e possibilitar a adequação da linguagem e do comportamento da equipe em campo. Após a coleta e análise dos dados, novas visitas foram feitas à comunidade, realizando-se reuniões com lideranças com o intuito de apresentar e discutir os resultados obtidos. Além destas atividades, a equipe foi surpreendida com reuniões organizadas pela própria comunidade em que houve participação ativa das mães e foram feitas sugestões de ações coletivas de promoção da saúde 


\section{Resultados e discussão}

\section{Características demográficas, ambientais e sócio-econômicas}

De acordo com a Tabela 1, a amostra estudada foi constituída por 100 crianças $(97,1 \%)$, das quais 47 eram do sexo masculino e 53, do feminino. A distribuição por faixa etária apresentou predomínio de crianças na faixa etária de 24 a 59 meses $(56,0 \%)$. A média de moradores por domicílio foi de 6,2 pessoas e a mediana, de 5,5 moradores.

Com relação à renda per capita familiar, $89,1 \%$ encontravam-se na faixa de 0 a 0,5 salário mínimo (sm) e $10,9 \%$ na faixa de 0,5 a 1,0 sm. Em todos os domicílios estudados, a renda per capita foi inferior a 1 s.m., revelando o baixo poder aquisitivo das famílias. Quanto à escolaridade, 6,3\% das mães eram sem instrução, $76,6 \%$ possuíam de 1 a 4 anos de estudo, 15,6\% de 5 a 8 anos e 1,6\% acima de 8 anos; dentre os pais, $1,7 \%$ eram sem instrução, $72,9 \%$ possuíam de 1 a 4 anos, $23,7 \%$ de 5 a 8 anos e 1,7\% acima de oito anos de escolaridade. Estudos demonstram que o nível de escolaridade dos pais é elemento decisivo para a percepção acerca dos cuidados com a criança, do aleitamento materno, do saneamento do meio, bem como para recorrer aos serviços de saúde em geral (IBGE, 1992).

Na análise das condições de saneamento ambiental, 100,0\% dos domicílios utilizavam água de poço artesiano; $89,1 \%$ queimavam ou enterravam o lixo e $10,9 \%$ jogavam ao redor do domicílio. No que concerne à existência de instalação sanitária, $50 \%$ possuíam sanitário de utilização exclusiva dos moradores do domicílio, 42,2\% tinham sanitários de utilização comum a mais de um domicílio e $7,8 \%$ não contavam com instalação sanitária. Com relação ao destino dos dejetos, $90,6 \%$ retidos em vala negra, 1,6\% em fossa séptica e 7,8\% espalhados pelo solo da mata circunvizinha. Domicílios com instalações sanitárias deficientes ou inexistentes provocam a transmissão de doenças infecciosas e parasitárias, agravando consequentemente as condições de saúde (Simões, 1992).

Nas moradias, $89,1 \%$ eram construídas com paredes de taquarussu e bacuri partidos ao meio; na cobertura, $73,4 \%$ de sapé ou folhas de palmeiras encontradas na região; no piso, $87,5 \%$ não possuíam revestimento (chão batido). Com relação ao número total de cômodos existentes na moradia, 93,8\% tinham de 1 a 3 cômodos. Quanto à presença de equipamentos domésticos, 39 domicílios possuíam fogão à gás $(60,9 \%)$, 34 com televisão $(53,1 \%), 33$ com rádio $(51,6 \%)$,
19 com aparelho de som $(29,7 \%), 17$ com geladeira $(26,6 \%)$ e 3 com freezer $(4,7 \%)$. O resultado obtido nessas variáveis evidenciam as más condições sócio-econômicas das famílias estudadas, nas quais as mudanças dependem fortemente da atuação do setor público, ou seja, através de políticas e investimentos sociais específicos para populações indígenas.

\section{Utilização de serviço de saúde materno-infantil}

Independente do número de consultas realizadas, a cobertura atingida pela assistência prénatal foi de $58,0 \%$, das quais $93,1 \%$ prestadas por serviços públicos. Se considerarmos mães assistidas pelo pré-natal somente quando tiverem acima de seis atendimentos durante o período de gestação, a cobertura passa a ser de $15,5 \%$. Este valor indica baixa cobertura e representa situação de risco para as gestantes indígenas e seus filhos. Com relação ao período gestacional em que iniciaram o pré-natal, $48,3 \%$ fizeram-no no primeiro trimestre, $32,8 \%$ no segundo e 18,9\% no terceiro. Estudo feito em Campo Grande, Mato Grosso do Sul, indicou que quase a totalidade das mulheres $(95,9 \%)$ realizou acompanhamento durante a gestação, o que demonstra a desigualdade dos cuidados oferecidos às gestantes nas diferentes regiões do Estado (Ribas et al., 1999).

Quanto à assistência ao parto, $40 \%$ teve parto domiciliar. A prevalência de partos operatórios foi de $8 \%$. Observou-se cobertura de $84 \%$ com relação ao cumprimento do esquema básico de vacinação em crianças, $12,8 \%$ estavam em atraso e $3,2 \%$ de crianças nunca haviam sido vacinadas.

O serviço de assistência à saúde presente na aldeia restringe-se a um técnico em enfermagem (FUNASA), uma agente de saúde (Secretaria Municipal de Saúde) e parteiras tradicionais. As parteiras tradicionais são reconhecidas pela comunidade, representando recursos humanos de grande valor para a saúde indígena. Essas parteiras não receberam treinamento, não são remuneradas e exercem a atividade com grande dificuldade - tal como: a falta de condições higiênicas, insumos básicos e transporte para uma situação de complicação obstétrica - fatores estes que elevam os riscos de mortalidade perinatal.

A unidade de saúde mais próxima fica aproximadamente a 30 quilômetros, com estrada sem pavimentação e linha de ônibus que disponibiliza uma unidade de transporte coletivo no período diurno, para atender usuários de três aldeias. Esses fatores, juntamente com 
a impossibilidade em se obter assistência segundo os próprios recursos, dificultam o acesso e a utilização dos serviços de saúde.

\section{Peso ao nascer}

Considerando as 47 crianças que tinham informações do peso ao nascer, foram observadas $17,0 \%$ com baixo peso (inferior a $2500 \mathrm{~g}$ ). O valor obtido representa elevada incidência de baixo peso ao nascer quando comparado com resultados da Pesquisa Nacional de Saúde e Nutrição (PNSN), que apresentou 9,6\% para crianças da região urbana e 12,3\% para área rural da Região Centro-Oeste (Monteiro, 1992). Estudo realizado em Campo Grande, Mato Grosso do Sul, identificou a incidência de 8,7\% (Ribas, 1997).

Esse resultado pode estar refletindo a qualidade de vida do grupo materno-infantil, representado pelas dificuldades sócio-econômicas da família, o acesso aos serviços de saúde e a baixa cobertura de assistência pré-natal, uma vez que o peso ao nascer pode ser determinado por fatores biológicos, sócio-econômicos e ambientais às quais a mulher está exposta durante a gestação. Estudos demonstram que crianças de baixo peso ao nascer são mais vulneráveis às condições ambientais e sociais desfavoráveis, apresentando maior risco de adoecer ou morrer (Almeida, 1994; Puffer \& Serrano, 1987). Segundo Monteiro (1992), uma das causas que provavelmente concorrem para aumentar a incidência de baixo peso ao nascer é a deficiência no atendimento pré-natal, pois exatamente os grupos populacionais identificados como sujeitos a maior risco de nascimentos de baixo peso são os que apresentam maior proporção de gestantes que não recebem adequada atenção pré-natal.

A incidência de baixo peso ao nascer observada - assim como a importância de ações preventivas - evidencia a necessidade imediata de atenção às mães indígenas durante a gravidez por meio de um programa que considere os valores culturais e as peculiaridades que envolvem concepção, gestação e nascimento em uma comunidade indígena.

\section{Estado nutricional}

A prevalência encontrada de déficits nutricionais foram de $8,0 \%$ para $\mathrm{P} / \mathrm{I}, 16,0 \%$ para $\mathrm{E} / \mathrm{I}$ e $1,0 \%$ para $\mathrm{P} / \mathrm{E}$ (Tabela 2 ). A proporção de déficits nutricionais com três escores $\mathrm{z}$ abaixo da mediana foi de $4,0 \%$. Esses valores demonstram elevada prevalência de desnutrição crônica e baixa prevalência de desnutrição aguda,
Tabela 1

Distribuição de freqüência de características

demográficas, sócio-econômicas e ambientais. Aldeia

Córrego do Meio, Mato Grosso do Sul, Brasil, 1999.

\begin{tabular}{|c|c|c|}
\hline Características & $n$ & $\%$ \\
\hline \multicolumn{3}{|l|}{ Sexo } \\
\hline Masculino & 47 & 47,0 \\
\hline Feminino & 53 & 53,0 \\
\hline \multicolumn{3}{|l|}{ Faixa etária } \\
\hline $0-5 \mathrm{~m}$ & 14 & 14,0 \\
\hline $6-11 \mathrm{~m}$ & 9 & 9,0 \\
\hline $12-23 m$ & 21 & 21,0 \\
\hline $24-59 m$ & 56 & 56,0 \\
\hline \multicolumn{3}{|l|}{ Número de moradores } \\
\hline $3-4$ & 21 & 32,8 \\
\hline $5-6$ & 24 & 37,5 \\
\hline $7-10$ & 19 & 29,7 \\
\hline \multicolumn{3}{|l|}{ Renda per capita familiar } \\
\hline $0 \vdash 0,5 \mathrm{s.m}$. & 57 & 89,1 \\
\hline $0,5 \vdash 1,0$ s.m. & 7 & 10,9 \\
\hline \multicolumn{3}{|l|}{ Escolaridade materna } \\
\hline sem escolaridade & 4 & 6,3 \\
\hline 1-4 anos & 49 & 76,6 \\
\hline 5-8 anos & 10 & 15,6 \\
\hline$>8$ anos & 1 & 1,6 \\
\hline \multicolumn{3}{|l|}{ Escolaridade paterna } \\
\hline sem escolaridade & 1 & 1,7 \\
\hline 1-4 anos & 43 & 72,9 \\
\hline $5-8$ anos & 14 & 23,7 \\
\hline$>8$ anos & 1 & 1,7 \\
\hline \multicolumn{3}{|l|}{ Água } \\
\hline Poço artesiano & 64 & 100,0 \\
\hline \multicolumn{3}{|l|}{ Lixo } \\
\hline Queimar/Enterrar & 57 & 89,1 \\
\hline Jogar ao redor & 7 & 10,9 \\
\hline \multicolumn{3}{|l|}{ Sanitário } \\
\hline Uso do domicílio & 32 & 50,0 \\
\hline Comum a mais domicílios & 27 & 42,2 \\
\hline Ausente & 5 & 7,8 \\
\hline \multicolumn{3}{|l|}{ Destino dos dejetos } \\
\hline Vala negra & 58 & 90,6 \\
\hline Fossa séptica & 1 & 1,6 \\
\hline Céu aberto & 5 & 7,8 \\
\hline \multicolumn{3}{|l|}{ Parede } \\
\hline Taquarussu/bacuri & 57 & 89,1 \\
\hline Alvenaria & 7 & 10,9 \\
\hline \multicolumn{3}{|l|}{ Cobertura } \\
\hline Sapé/Bacuri & 47 & 73,4 \\
\hline Telha & 17 & 26,6 \\
\hline \multicolumn{3}{|l|}{ Piso } \\
\hline Chão batido & 56 & 87,5 \\
\hline Cimentado & 7 & 10,9 \\
\hline Cerâmico & 1 & 1,6 \\
\hline \multicolumn{3}{|l|}{ Número de cômodos } \\
\hline 1 a 3 & 60 & 93,8 \\
\hline 4 а 6 & 4 & 6,2 \\
\hline
\end{tabular}


Tabela 2

Distribuição de freqüência de déficits nutricionais pelos índices $P / I, E / I, P / E$ e de obesidade pelo índice P/E segundo sexo. Aldeia Córrego do Meio, Mato Grosso do Sul, Brasil 1999.

\begin{tabular}{|c|c|c|c|c|c|}
\hline \multirow[t]{2}{*}{ Sexo } & \multirow[t]{2}{*}{$n$} & \multicolumn{3}{|c|}{ Déficits nutricionais } & \multirow{2}{*}{$\begin{array}{c}\text { Obesidade } \\
\qquad \begin{array}{c}P / E \% \\
>+2 z\end{array}\end{array}$} \\
\hline & & $\begin{array}{l}P / I \% \\
<-2 z\end{array}$ & $\begin{array}{l}E / I \% \\
<-2 z\end{array}$ & $\begin{array}{c}P / E \% \\
<-2 z\end{array}$ & \\
\hline Masculino & 47 & 10,6 & 21,3 & - & 6,4 \\
\hline Feminino & 53 & 5,7 & 11,3 & 1,9 & 3,8 \\
\hline Total & 100 & 8,0 & 16,0 & 1,0 & 5,0 \\
\hline
\end{tabular}

mas é importante lembrar que estudos de observação transversal não são adequados para aferição de prevalência de desnutrição aguda. $\mathrm{Na}$ análise segundo o sexo, para o índice $\mathrm{P} / \mathrm{I}$ foram encontrados $10,6 \%$ para meninos e $5,7 \%$ para meninas; no índice E/I, $21,3 \%$ para meninos e $11,3 \%$ para meninas - o que representa maior gravidade da situação entre os meninos - e, para P/E, nenhum caso entre os meninos e $1,9 \%$ para meninas.

A distribuição de freqüência de obesidade foi de $5 \%$, dos quais $6,4 \%$ no sexo masculino e $3,8 \%$ no feminino. A razão entre a prevalência de desnutrição e a obesidade infantil foi acima de três desnutridos para um obeso. Em estudo realizado em comunidade indígena urbana, observou-se a prevalência de $6,3 \%$ de obesidade, sendo $3,7 \%$ no sexo masculino e $8,3 \%$ no feminino (Ribas et al., 1999).

Para qualificar a prevalência de crianças com retardo severo de crescimento, estabeleceu-se a comparação com estimativas da PNSN e da Pesquisa Nacional de Demografia e Saúde (PNDS). Nesta análise, a prevalência de retardo de crescimento na população indígena é superior à encontrada na população brasileira como um todo, provavelmente refletindo as condições sócio-econômicas de populações indígenas representadas pela baixa renda familiar, carência de alimentos, falta de atenção à saúde, precárias condições de saneamento e baixa escolaridade dos pais. Segundo a PNSN (1989, apud Monteiro, 1992), a prevalência de retardo de crescimento linear infantil na área rural da região Centro-Oeste era $10,2 \%$, valor inferior ao observado neste estudo.

Em comunidade indígena Teréna localizada no perímetro urbano do Município de Campo Grande, Mato Grosso do Sul, e contando com projetos de assistência à saúde, geração de renda e saneamento básico, a prevalência encontrada foi de $9,5 \%$ de retardo de crescimento (Ribas et al., 1999). Esta comparação poderia indicar a possível ação de medidas preventivas e assistenciais sobre as condições nutricionais de crianças indígenas.

Comparando com estudos realizados em outras comunidades indígenas, a prevalência de desnutrição infantil observada é inferior às reportadas por grupos indígenas da região amazônica. Em 1987, entre crianças Suruí (RO) menores de cinco anos, a prevalência de retardo de crescimento infantil era de 50,6\% (Coimbra Jr. \& Santos, 1991). Estudo realizado em crianças Parakanã (PA) observou 53,5\% em 1989, 48,1\% em 1990 e 50,6\% em 1991 (Martins \& Menezes, 1994). Entre os Xavánte (MT), na avaliação nutricional de crianças com 0 a 4 anos, foi encontrada a prevalência de $27,7 \%$ no ano de 1994 e 22,0\% em 1995 (Gugelmim, 1995).

A Tabela 3 apresenta a distribuição de freqüência e percentual de retardo de crescimento segundo a faixa etária, em que de 0 a 5 meses foi encontrado $7,1 \%$, representada por uma criança que nasceu com baixo peso e foi desmamada ainda nos primeiros dias de vida. Na faixa etária de 6 a 11 meses foram observados os maiores valores de déficit de crescimento linear, período de grande vulnerabilidade em razão da introdução de novos alimentos e início da dependência dos suprimentos alimentares do domicílio. Nas crianças de 12 a 23 meses de idade, $26,3 \%$ eram de déficit e nas de 24 a 59 meses, foi de $13,7 \%$. Tais resultados indicam que ações de recuperação nutricional deveriam ser rapidamente incorporadas à programação de assistência à saúde indígena, considerando que poderá haver reversibilidade do déficit estatural em crianças com déficit de crescimento linear se houver condições adequadas de recuperação, com atendimento que contemple os diversos aspectos envolvidos.

Com relação à renda per capita, a menor faixa de renda (0 a 0,5 s.m) apresentou a prevalência de 15,0\% e, a maior (0,5 a 1,0 sm), 1,0\%. Mesmo com valores inferiores a $1 \mathrm{sm}$ per capita para todos os domicílios, foi possível observar diferenças, evidenciando a ação da renda sobre o estado nutricional infantil.

\section{Consumo alimentar}

Na análise do consumo alimentar, participaram 98 crianças menores de cinco anos. Apesar das refeições não serem realizadas em horários rígidos, foram observadas três refeições relatadas como principais, distribuídas ao longo do dia, sendo a primeira, ao acordar no nascer do sol; a segunda, no meio do dia e a terceira, no início da noite, quando o sol se põe. Nos intervalos há consumo de frutos coletados ao redor 
do domicílio ou tubérculos - mandioca e/ou batata - cozidos ou assados. O arroz é o alimento mais freqüente e em maior quantidade nas refeições, constituindo, muitas vezes, o único alimento. Na seqüência estão presentes a mandioca, feijão, macarrão e a carne. A carne é bastante valorizada na refeição, mas de difícil acesso para a maioria das famílias e, quando presente, a utilizada é a de menor custo, com elevado teor de gordura. Com relação à criação de animais, $81,3 \%$ das famílias criam galinhas e/ou porcos em pequena escala, o que leva a consumo reduzido, reservado apenas para ocasiões especiais. Quanto à forma de preparo das carnes, há predomínio de frituras. A qualidade da dieta está condicionada à disponibilidade, acesso e, em especial, ao custo dos alimentos. A dieta das famílias é composta basicamente por alimentos de menor custo, como o arroz, mandioca, açúcar e carnes de elevado teor de gordura.

Os Teréna se autodenominam pokée - que significa terra - e são essencialmente agricultores, pois $89,1 \%$ das famílias contam com plantação de roças e pomar ao redor dos domicílios. Relato de lideranças referem que, no passado, a agricultura, a caça, a pesca e a coleta de frutos e raízes eram as atividades de subsistência, mas, no presente, há necessidade da busca de trabalho remunerado nas fazendas e municípios vizinhos à aldeia - em particular, em virtude da redução territorial, da degradação dos recursos naturais e do crescimento populacional, o que por si só, justifica a importância de garantir o direito às áreas com extensão adequada.

Nas roças foi identificado basicamente o cultivo de arroz, feijão, mandioca e milho, mas, em algumas delas, também de batata doce, abóbora, quiabo, abacaxi, banana, melancia e cana de açúcar, variando de acordo com a área e a época do ano. Cada família possui área disponível para plantar, variando de 1 a 10 hectares, com predomínio de áreas menores. Na última safra, $72,6 \%$ destinaram a produção exclusivamente para o consumo da própria família, 6,5\% distribuíram entre outras famílias e $21,0 \%$ puderam comercializar os produtos. A diversidade de produtos cultivados não garante o seu consumo adequado seja pela quantidade produzida ou pela ausência de sistema de armazenamento e conservação dos produtos. Os pomares não apresentam demarcações, permitindo livre acesso a qualquer integrante da aldeia que queira coletar frutos, tendo-se observado o consumo de manga, mamão e goiaba em alguns meses do período compreendido pelo estudo. Outras árvores frutíferas -
Tabela 3

Distribuição de freqüência de retardo de crescimento segundo faixa etária.

Aldeia Córrego do Meio, Mato Grosso do Sul, Brasil 1999.

\begin{tabular}{lcc}
\hline Faixa etária (meses) & \multicolumn{2}{c}{ Retardo de Crescimento E/I } \\
Freqüência & Percentual \\
\hline $0-5$ & 1 & 7,1 \\
$6-11$ & 3 & 33,3 \\
$12-23$ & 5 & 23,8 \\
$24-59$ & 7 & 12,5 \\
Total & 16 & 16,0 \\
\hline
\end{tabular}

como: limão, laranja, tangerina, tamarindo e caju - foram identificadas, mas sem produção de frutos no período.

Com relação à pesca, 35,9\% realizam esta atividade esporadicamente, $15,6 \%$ caçam e $67,2 \%$ coletam frutos silvestres. A captura de animais e coleta de frutos silvestres é feita com dificuldades devido à limitação da área na reserva. Os animais apreendidos com maior freqüência são o tatu, capivara, nambu, paca e porco do mato. Dentre os frutos coletados foram identificados a bocaiúva (Acrocomia aculeata), araticum (Annona dioica), jatobá (Hymenaea stigonocarpa), jenipapo (Genipa americana), coroa (Mouriri elliptica), buriti (Mauritia vinifera), pequi (Caryocar brasiliense), jurubeba (Solanum paniculatum) ingá (Inga uruguensis), guariroba (Syagrus oleracea), araçá (Psidium guineense), urucum (Bixa orellana) e caraguatá (Bromelia balansae).

Quanto aos produtos industrializados consumidos foram identificados o açúcar, sal, óleo de soja, macarrão, café, farinha de trigo, leite em pó, extrato de tomate, refresco em pó, chá, refrigerante, lingüiça, bala, pães e biscoitos. Esses produtos estão presentes principalmente na dieta das famílias que dispõem de aposentadoria ou salário, as quais os adquirem no período de recebimento. O predomínio de alimentos ricos em carboidratos - açúcar, macarrão, refrigerantes, balas, pães e biscoitos - e em gorduras de origem animal - carnes gordas -, associado à pouca atividade física poderia estar favorecendo o aparecimento de obesidade entre as mães, uma vez que as atividades que demandam maior gasto energético - como as ocupações agrícolas, a caça e a pesca - são atividades eminentemente masculinas.

A Tabela 4 apresenta a análise da dieta segundo adequação percentual média dos nutrientes que a compõem. O consumo de energia, retinol, cálcio e ferro não atingem as reco- 
Adequação percentual média do consumo de energia, proteína, retinol, cálcio, vitamina $C$ e ferro segundo faixa etária. Aldeia Córrego do Meio, Mato Grosso do Sul, 1999.

\begin{tabular}{lrcccccc}
\hline Faixa etária & $\mathbf{n}$ & Energia \% & Proteína \% & Retinol \% & Cálcio \% & Vitamina C \% & Ferro \% \\
\hline 0-5 m & 13 & 64,6 & 71,5 & 58,9 & 79,1 & 62,5 & 8,4 \\
$6-11 \mathrm{~m}$ & 9 & 52,3 & 61,7 & 59,4 & 23,8 & 63,5 & 14,1 \\
$12-23 \mathrm{~m}$ & 20 & 55,9 & 97,3 & 47,4 & 23,9 & 76,3 & 33,0 \\
$24-59 \mathrm{~m}$ & 56 & 55,1 & 136,0 & 48,5 & 23,9 & 98,2 & 60,4 \\
\hline
\end{tabular}

mendações nas diferentes faixas etárias. O consumo de proteína é insuficiente na dieta das crianças menores de um ano, enquanto naquelas de 1 a 2 anos apresenta valores que atingem as recomendações e nas acima de dois anos, ultrapassa as recomendações. O consumo de vitamina $\mathrm{C}$ somente atinge as recomendações na dieta das crianças acima de dois anos, representado especialmente pelo consumo de goiaba nas refeições intermediárias.

$\mathrm{Na}$ análise do consumo energético total, $91,8 \%$ das dietas foram classificadas como insuficientes, $7,2 \%$ como adequadas e $1,0 \%$ como excessivas. Com relação ao consumo energético, foi tido como insuficiente de acordo com as faixas etárias: $84,6 \%$ na dieta das crianças de 0 a 5 meses, $100 \%$ de 6 a 11 meses, $95 \%$ de 12 a 23 meses e $91,1 \%$ acima de dois anos. A prevalência de déficit de estatura para idade segundo o consumo energético evidenciou 15,0\% de retardo de crescimento nas crianças com dietas insuficientes e 1,0\% entre as adequadas.

\section{Considerações finais}

A prevalência de desnutrição infantil é elevada, superior à identificada em comunidade indígena urbana e área rural da região Centro-Oeste, mas inferior às reportadas por grupos indígenas da região amazônica. $\mathrm{O}$ estado nutricional mostrou-se influenciado pela renda, sexo e faixa etária, apresentando maior freqüência de retardo de crescimento quando pertenciam a família de menor renda per capita, ao sexo masculino e na faixa etária de 6 a 23 meses de idade.

A precária condição sócio-econômica das famílias é representada pela alta densidade familiar, baixa renda, baixa escolaridade dos pais, difícil acesso a serviços de saúde e condições domiciliares insalubres.

A cobertura atingida pela assistência prénatal é baixa, agravada pelo número de partos domiciliares. Os dados referentes a peso ao nascer revelam elevada incidência de baixo peso, considerada situação como grave com relação a valores nacionais e internacionais. A cobertura vacinal é satisfatória, mas necessita ser ampliada, tomando-se em conta a importância de uma série completa de vacinas.

A análise da adequação percentual média dos nutrientes que compõem a dieta infantil indica o não atendimento às recomendações nutricionais nas diferentes faixas etárias; no que diz respeito ao consumo energético total, quase a totalidade das dietas foi classificada como insuficiente.

Esses fatores somados ao processo de envolvimento com a vida urbana, próxima das aldeias, contribuem para a simultaneidade de problemas que caracterizam a atual condição de vida destas famílias, apontando a necessidade de implementação imediata de política social específica para populações indígenas, com programas que melhorem a assistência à saúde, ao saneamento básico, à educação e ao acesso à terra. 


\section{Agradecimentos}

À Fundação Nacional do Índio pela autorização de ingresso e pelo apoio logístico na terra indígena.

Aos acadêmicos que participaram ativamente de todas as fases da pesquisa e que foram fundamentais para a concretização deste trabalho: Silvia Fuzetto, Rita de Cássia Ribeiro, Sandra Arteaga, Marcio Belini e Mariane Leles.

À guia indígena pela valiosa colaboração no acesso às famílias: Eliane Dias.

À Fundação de Apoio ao Desenvolvimento do Ensino, Ciência e Tecnologia do Estado de Mato Grosso do Sul pelo financiamento recebido (FUNDECT/0017/00).

\section{Referências}

ALMEIDA, M. F., 1994. Mortalidade Neonatal em Santo André, São Paulo. Tese de Doutorado, São Paulo: Faculdade de Saúde Pública, Universidade de São Paulo.

COIMBRA Jr., C. E. A. \& SANTOS, R. V., 1991. Avaliação do estado nutricional num contexto de mudança sócio-econômica: O grupo indígena Suruí do Estado de Rondônia, Brasil. Cadernos de Saúde Pública, 7:538-562.

GADELHA, R., 1982. As Missões Jesuíticas do Itatim: Estruturas Sócio-econômicas do Paraguai Colonial nos Séculos VI e XVII. Rio de Janeiro: Paz e Terra.

GUGELMIM, S. A., 1995. Nutrição e Alocação de Tempo dos Xavánte de Pimentel Barbosa, Mato Grosso: Um Estudo em Ecologia Humana e Mudanças. Dissertação de Mestrado, Rio de Janeiro: Escola Nacional de Saúde Pública, Fundação Oswaldo Cruz.

IBGE (Fundação Instituto Brasileiro de Geografia e Estatística), 1992. Perfil Estatístico de Crianças e Mães no Brasil. Aspectos de Saúde e Nutrição no Brasil 1989. Rio de Janeiro: IBGE.

LOHMAN, T. G.; ROCHE, A. F. \& MARTORELL, R., 1988. Anthropometric Standardization Reference Manual. Illinois: Human Kinetics.

MARTINS, S. J. \& MENEZES, R. C., 1994. Evolução do estado nutricional de menores de 5 anos em aldeias indígenas da Tribo Parakanã, na Amazônia Oriental Brasileira (1989-1991). Revista de Saúde Pública, 28:1-8.

MONTEIRO, M. F. G., 1992. Baixo peso ao nascer. In: Perfil Estatístico de Crianças e Mães no Brasil. Aspectos de Saúde e Nutrição no Brasil 1989 (Fundação Instituto Brasileiro de Geografia e Estatística, ed.), pp. 11-18, Rio de Janeiro: IBGE.

NCHS (National Center for Health Statistics), 1978. Growth Curves for Children, Birth-18 years. Department of Health, Education and Welfare, Publication PHS 78-1650. Washington, DC: NCHS.
NRC (National Research Council), 1989. Recommended Dietary Allowances. Washington, DC: National Academy Press.

OBERG, K., 1949. The Terena and the Caduveo of Southern Mato Grosso, Brazil. Washington, DC: Institute of Social Anthropology.

PHILIPPI, S. T.; SZARFARC, S. C. \& LATTERZA, A. R., 1996. Virtual Nutri. Versão 1.0 for Windows. São Paulo: Departamento de Nutrição, Faculdade de Saúde Pública, Universidade de São Paulo.

PUFFER, R. R. \& SERRANO, C. V., 1987. Patterns of Birthweights. PAHO Scientific Publication 504. Washington, DC: Pan-American Health Organization.

RIBAS, D. L. B., 1997. Avaliação Nutricional de Crianças Menores de Cinco Anos de Idade no Município de Campo Grande MS, Brasil. Dissertação de Mestrado, Campo Grande: Universidade Federal de Mato Grosso do Sul.

RIBAS, D. L. B.; ZORZATTO, J. R. \& PHILIPPI, S. T., 1999. Nutrição de crianças indígenas. In: Congresso Latino-Americano de Nutrição Humana; Resumos, p. 120, Gramado: Conselho Regional de Nutricionistas.

SILVA, F. A., 1949. Mudança cultural dos Terena. Revista do Museu Paulista, 3:271-280.

SIMÕES, C. C., 1992. O estudo dos diferenciais na mortalidade infantil segundo algumas características sócio-econômicas. In: Perfil Estatístico de Crianças e Mães no Brasil. Aspectos de Saúde e Nutrição no Brasil 1989 (Fundação Instituto Brasileiro de Geografia e Estatística, org.), pp. 61-78, Rio de Janeiro: IBGE.

WHO (World Health Organization), 1995. Physical Status: The Use and Interpretation of Anthropometry. WHO Technical Report Series 854. Geneva: WHO. 